\title{
An Emerging Treatment Alternative for Anemia in CKD Patients: A Review on Daprodustat
}

\section{Becker $\mathrm{K}^{1^{*}}$ and Jones $\mathrm{JJ}^{2}$}

${ }^{1}$ Department of Pharmacy, Lerin Drug Co Inc, New York, USA

${ }^{2}$ Department of Pharmacy, St. John's University, Jamaica, New York, USA

*Corresponding author: Kimberly Becker, Department of Pharmacy, Lerin Drug Co Inc, NY-10025, USA, Tel: 212 678 0636; E-mail: kimberly.anne.becker@gmail.com

Received date: Oct 9, 2017; Accepted date: Oct 24, 2017; Published date: Oct 30, 2017

Copyright: ( 2017 , Becker K, et al. This is an open-access article distributed under the terms of the Creative Commons Attribution License, which permits unrestricted use, distribution, and reproduction in any medium, provided the original author and source are credited.

\begin{abstract}
This review article informs the reader about daprodustat (GSK1278863), a novel oral therapeutic agent for the treatment of anemia currently in development by GlaxoSmithKline, inclusive of published and ongoing clinical trial information. Results from phase II studies have demonstrated a promising therapeutic alternative for chronic kidney disease (CKD) patients that may ultimately change future clinical practice.
\end{abstract}

Keywords: Daprodustat; Anemia; HIF-inhibitor; HIF; Chronic kidney disease

\section{Introduction}

Greater than 1 in 7 Americans are estimated to be living with chronic kidney disease (CKD) [1]. Patients with CKD are prone to developing multiple comorbidities, including anemia [2]. As the disease state progresses, patients are at an increased risk of developing anemia. Anemia in CKD patients may be caused by iron deficiency, inflammation, or a disruption in the production of erythropoietin (EPO) [2,3]. Erythropoietin is a hormone that stimulates the production of red blood cells [4]. Deficiencies of this hormone are currently managed with erythropoietin stimulating agents [ESAs], usually in combination with iron supplementation. However, the FDA has issued warnings on the use of EPO analogs due to risk of death and serious cardiovascular events [5-7]. The cardiovascular safety findings of EPO analogs were based on a number of controlled trials which targeted hemoglobin $(\mathrm{Hb})$ values greater than $11 \mathrm{~g} / \mathrm{dL}[8,9]$. The warnings stated that no trial has identified a $\mathrm{Hb}$ target level, ESA dose, or dosing strategy that does not increase these risks. The FDA labeling recommends using the lowest epoetin alfa dose sufficient to reduce the need for red blood cell (RBC) transfusions [5-7]. Daprodustat is an orally administered, small molecule hypoxia-inducible factor prolyl hydroxylase (HIF-PH) inhibitor currently in phase III development [10]. GlaxoSmithKline expects the completion of their phase III trials in 2018 and 2019 for US approval [11]. This agent offers a novel approach to managing anemia in CKD patients that could affect clinical practice recommendations and guidelines. This article is based on previously conducted studies and does not involve any new studies of human or animal subjects performed by the authors.

\section{Scientific Summary}

\section{Mechanism of action}

When the body is exposed to low oxygen levels, such as at increased altitudes the body stimulates the production of EPO to increase RBCs and restore oxygen balance $[3,12]$. This mechanism of compensation is initiated through transcription factor, HIF- $\alpha$, more specifically the isoform HIF-1 $\alpha$ which is commonly referred to as HIF-1. When HIF- $\alpha$ accumulates it forms a functional dimer with HIF- $\beta$, which subsequently translocates to the nucleus where it binds to hypoxia response elements (HREs) on DNA. This results in the induction of gene expression of EPO, which in turn promotes an erythropoietic response and up regulation of iron transport $[3,13]$. When the body has adequate oxygen levels, HIF- $\alpha$ is degraded and regulated by HIFPH. Daprodustat stabilizes HIF- $1 \alpha$ by inhibiting HIF-PH, therefore increasing levels of HIF- $\alpha$, leading to increases in EPO, and decreasing hepcidin, an indirect regulator of iron absorption and utilization $[3,12,13]$.

\section{Pharmacokinetics (PK)}

Daprodustat is a highly protein bound molecule in vivo and undergoes oxidative metabolism primarily in the liver by the CYP2C8 enzyme into 6 metabolites with an approximate half-life of up to 4 hours (100 mg dose) [13]. Daprodustat most likely undergoes first-pass metabolism in the liver and possibly the gut wall.

Results from a study designed to characterize differences in PK/PD parameters between Japanese and Caucasian participants revealed rapid absorption of daprodustat with a median concentration peak time $\left(\mathrm{t}_{\max }\right)$ between 1.3 to 2.5 hours and 1.0 to 2.0 hours in the Japanese group and Caucasian group, respectively (NCT02348372) [14]. Across the 10, 25, 50 and $100 \mathrm{mg}$ doses, the mean terminal halflife $\left(\mathrm{t}_{1 / 2}\right)$ ranged from 1.1 to 2.3 hours and 0.9 to 1.9 hours, respectively [14]. Suggestively, higher exposure as determined by Cmax and AUCinf was observed in the Japanese group relative to the Caucasian group [14]. With regard to food, co-administration of a single dose of daprodustat with a high-fat/high calorie meal did not significantly change its plasma AUC; however, a mean reduction of $29 \%$ was observed for the Cmax (NCT01376232) [13]. It is unknown whether this will translate into clinical relevance; thus, in order to minimize drug exposure variability within patients, investigators recommended consistent administration of daprodustat either with food or without food. Concomitant administration with steady-state gemfibrozil, a strong CYP2C8 inhibitor, resulted in significantly increased AUCinf, 
Page 2 of 5

Cmax, and $t_{1 / 2}$ of daprodustat, which suggests significant drug-drug interactions would occur with CYP2C8 inhibitors [13].

\section{Pharmacodynamics (PD)}

Daprodustat demonstrated an appreciable dose-dependent increase in a variety of pharmacodynamic markers, including EPO concentration, absolute reticulocyte count, $\mathrm{Hb}$ level, total iron-binding capacity (TIBC), and unsaturated iron-binding capacity in target populations [15,16]. Conversely, daprodustat demonstrated a dosedependent decrease in hepcidin concentration with a subsequent trend towards baseline during follow-up periods [15,16]. Additionally, daprodustat decreased the concentration of ferritin at all doses, which signifies utilization of iron stores [15,16]. Surrogate markers of pharmacodynamic effects, including EPO, VEGF, and absolute reticulocyte counts, have also exhibited dose-ordered increases over a range of 10 to $100 \mathrm{mg}$ in both Japanese and Caucasian ethnic populations [14].

\section{Preclinical data}

Biochemical in vitro characterization of GSK1278863 revealed timedependent, reversible inhibition against prolyl hydroxylase 3 (PHD3) [17]. Under experimental conditions, the residence half-life value was calculated to be $13-19 \min (n=4)$ for PHD3, indicating that GSK1278863 does not dissociate instantaneously from the PHD3 enzyme. Pharmacological activity in vivo demonstrated peak circulating plasma EPO protein levels at 12 hours post-dose for female B6D2F1 mice $(n=6)$ administered a single dose of GSK1278863 at 60 $\mathrm{mg} / \mathrm{kg}$ relative to vehicle-treated mice with minimal impact on VEGF concentrations. In addition, statistically significant increases in reticulocytes, red blood cell counts, hemoglobin, and hematocrit were observed in compound-treated mice after dosing with 3, 10 and 30 $\mathrm{mg} / \mathrm{kg} /$ day. In chronic oral toxicity studies in rats, generalized vascular congestion, thrombosis, and/or multi-organ pathology were the doselimiting effects of GSK1278863 due to increases in hematocrit [17]. During the compound selection criteria, GSK1278863 was discovered as a result of prioritizing selectivity for PHD over collagen prolyl hydroxylase $(\mathrm{CP} 4 \mathrm{H})$ in order to mitigate the risk of valvulopathy observed with structurally similar chemical compounds. Unlike the non-selective HIF-prolyl-hydroxylase inhibitors, GSK1278863 did not cause cardiac valve lesions in 14 or 28 day rodent oral toxicity studies or in $\mathrm{dog} /$ monkey studies of up to 9 months on maximum tolerated doses. Thus, GSK1278863 demonstrated an optimized biological profile and pharmaceutical properties acceptable to support progression into clinical trials.

\section{Phase I trials}

The safety and tolerability of repeat doses of daprodustat in healthy adult participants have been evaluated in several phase I studies [11]. Specifically NCT00750256, which evaluated doses from $2 \mathrm{mg}$ to 450 mg with 6 cohorts; NCT00840320 evaluated 15, 25, 50, and $150 \mathrm{mg}$ doses; NCT01406340 assessed a dose of $5 \mathrm{mg}$ in a mixed population of healthy and renally impaired participants; and NCT02293148 evaluated effects on cardiac repolarization with a $500 \mathrm{mg}$ dose.

An ongoing phase I study, NCT02243306, is examining the PK of 5 $\mathrm{mg}$ daprodustat and metabolites in a targeted patient population, i.e., participants with end stage renal disease (ESRD) undergoing peritoneal dialysis (PD) [11].

\section{Phase II therapeutic trials}

\author{
Management of anemia in hemodialysis (HD) patients: 4, 6, 8 and \\ $10 \mathrm{mg}$
}

A phase II, randomized, placebo-controlled, dose ranging study of daprodustat was conducted in Japanese patients on HD (NCT0201971) [18]. In a phase I trial of daprodustat conducted in healthy volunteers, Japanese patients had a 1.3 to 1.5 fold higher plasma drug concentration [14]. This study sought to evaluate the $\mathrm{Hb}$ dose response and safety of daprodustat in Japanese participants $[14,18]$. The study randomized 97 Japanese participants who had been on HD for $\geq 8$ weeks and who were effectively managed with an ESA resulting in stable $\mathrm{Hb}$ levels. Participants discontinued their ESA for 2-8 weeks prior to enrollment; if participants had a decrease in $\mathrm{Hb}$ of $\geq 0.5 \mathrm{~g} / \mathrm{dL}$ with a resulting baseline of $8.5-10 \mathrm{~g} / \mathrm{dL}$, they were eligible for inclusion. Patients were randomized $1: 1: 1: 1: 1$ to receive $4,6,8,10 \mathrm{mg}$ of daprodustat or placebo daily. Eighty-nine percent of patients completed the trial with the mean baseline $\mathrm{Hb}$ ranging from 9.68 to $9.92 \mathrm{~g} / \mathrm{dL}$. The primary endpoint of change in Hb levels at week 4 was $0.28,-0.01,0.54,0.97$ in the $4,6,8,10 \mathrm{mg}$ groups, respectively, compared to a decrease of $1.41 \mathrm{~g} / \mathrm{dL}$ in the placebo arm. Changes in other blood parameters such as red blood cell count (RBC) and reticulocytes were consistent with the changes in Hb. Plasma EPO concentrations increased in a dose related manner up to $8 \mathrm{mg}$, with no additional increases with $10 \mathrm{mg}$. HIF-1 upregulates VEGF, which is implicated in angiogenic activity, tumor growth, and macular edema. VEGF levels were also measured and there were no significant differences observed between any of the groups.

Other secondary endpoints included iron parameters [18]. A total of $5 \%$ and $20 \%$ of participants received stable oral or IV iron supplementation throughout the study. There was a dose dependent decrease in hepcidin and ferritin coupled with increases in transferrin and TIBC, indicating a dose response relationship with increased iron utilization. Modest decreases in lipid levels were seen at week 4 in the daprodustat arm and there was a higher incidence of nasopharyngitis in participants who received daprodustat compared to placebo.

\section{Management of anemia in non-dialysis dependent (NDD) \& HD CKD patients}

Studies 1 and 2: 0.5, 2 and $5 \mathrm{mg}$ : Two additional phase II A randomized, blinded, controlled studies were conducted with daprodustat over a 4 week time period in separate populations with CKD to further evaluate the safety and efficacy of daprodustat [16]. The first study, referred to as the 'nondialysis study' was conducted in participants with CKD stages 3-5 not undergoing dialysis who had not used recombinant human erythropoietin (rhEPO) within the past 7 weeks (NCT01587898). The second study, referred to as the 'Hemodialysis Study' (HDD), was conducted in participants receiving hemodialysis three times weekly and adequately responding to rhEPO treatment (NCT01587924). These participants were either switched from rhEPO to daprodustat or maintained on rhEPO during the course of the study. Per the protocol, participants would be withdrawn from the studies if they their hemoglobin was $<8.0 \mathrm{~g} / \mathrm{dL}, 13.0 \mathrm{~g} / \mathrm{dL}$ or they experienced a change of $2.0 \mathrm{~g} / \mathrm{dL}$ in a one-week time period. Participants were randomized $1: 1: 1: 1$ in each study with the active treatment being one of three doses of daprodustat, i.e., $0.5 \mathrm{mg}, 2 \mathrm{mg}$ or $5 \mathrm{mg}$; placebo was used in the non-dialysis study while rhEPO was used in the HDD study. A follow-up period of 2 weeks applied to each study. 
Seventy three participants were randomized in the non-dialysis study, of which $59(81 \%)$ had completed the study through follow-up at week 6 and 54 had received daprodustat [15]. Considering demographic characteristics, $100 \%$ of the non-dialysis participants had cardiovascular risk factors at baseline, including hypertension (97\%), hyperlipidemia (76\%), and diabetes (69\%). In the HDD study, 83 participants were randomized, of which 70 (84\%) participants had completed the study through follow-up and 62 had received daprodustat. Similarly, $95 \%$ of the dialysis participants had cardiovascular risk factors, with the leading characteristic of hypertension (95\%) followed by hyperlipidemia (57\%) and diabetes (45\%). Over the 4 week treatment period, the mean SD observed change from baseline (CFB) in hemoglobin for the $0.5,2$ and $5 \mathrm{mg}$ doses of daprodustat were $-0.121 \quad(n=11), 0.121 \quad(n=15), 0.951 \mathrm{~g} / \mathrm{dL}$ $(\mathrm{n}=17)$ in the non-dialysis study, respectively; in the HDD study the hemoglobin results for the $0.5,2$ and $5 \mathrm{mg}$ doses of daprodustat were $-1.06 \quad(n=18), \quad-0.93 \quad(n=18), \quad-0.08 \quad \mathrm{~g} / \mathrm{dL} \quad(\mathrm{n}=17)$, respectively. Additionally, the observed 4 week CFB in hemoglobin for rhEPO in HDD study was $-0.25 \mathrm{~g} / \mathrm{dL} \quad(\mathrm{n}=19)$. EPO concentrations did not accumulate over the treatment period with daprodustat. Comparing the median peak EPO concentrations across the groups in the HDD study, the results were $424.9,13.9,12.7$, and $24.7 \mathrm{U} / \mathrm{L}$ for the rhEPO, $0.5 \mathrm{mg}, 2 \mathrm{mg}$ and $5 \mathrm{mg}$ of daprodustat groups, respectively. There were no clinically relevant changes in plasma VEGF in both daprodustat studies. The pharmacodynamic assessments were limited to 11 hours post-dose and thus the true peak plasma concentrations of EPO and VEGF may not have been captured. Lastly, few safety events occurred in these two trials with a total of 5 AEs reported for 1 or more participants receiving daprodustat; 2 experienced nausea in the nondialysis study and 3 experienced anemia in the HDD study. However, drug-related adverse events were not clearly distinguished in these studies. While no deaths occurred in either study, two serious AEs were reported post-therapy in the HDD study for participants receiving $0.5 \mathrm{mg}$ of daprodustat, which included an abnormal liver function test and acute respiratory failure.

Study 3: 10, 25, 50 and $100 \mathrm{mg}$ : A phase II A, randomized, placebocontrolled, dose ranging study of daprodustat was conducted in anemic patients $(\mathrm{Hb} \leq 11.0 \mathrm{~g} / \mathrm{dL}$ ) with CKD (NCT1047397) [15]. Participants were grouped separately according to degree of renal impairment; that is either NDD-CKD stages 3-5 or hemodialysisdependent CKD stage 5 (corresponding eGFR: 15 to $59 \mathrm{~mL} / \mathrm{min} / 1.73$ $\mathrm{m}^{2}$ for stages $3-4 ; 10$ to $<15 \mathrm{~mL} / \mathrm{min} / 1.73 \mathrm{~m}^{2}$ for stage 5 ). The study evaluated safety and tolerability end points as well as collected PK/PD data of once-daily administration of daprodustat over a period of 28 days with post-treatment follow-up to Day 57. Participants were enrolled to receive doses of $10,25,50$ and $100 \mathrm{mg}$ in the non-dialysis group (CKD-3/4/5) and doses of 10 and $25 \mathrm{mg}$ in the hemodialysis group (CKD-5D). A total of 107 participants, predominantly white females and patients of Asian descent, were enrolled in this study. Seventy participants were randomized to the CKD-3/4/5 group and 37 randomized to the CKD-5D group, of which 61 and 31 participants received active treatment, respectively.

A priori response rates were defined as a $\mathrm{Hb}$ level increase of 1.0 $\mathrm{g} / \mathrm{dL}$ and $0.5 \mathrm{~g} / \mathrm{dL}$ for the CKD-3/4/5 and CKD-5D groups, respectively [15]. The percentage of participants in the groups achieving these response were $63 \%$ and $91 \%$ for the CKD-3/4/5 group and $71 \%$ and $89 \%$ for the CKD-5D group. Per the protocol defined safety criteria, dosing in any treatment arm would be stopped if the $\mathrm{Hb}$ level was $>13.5 \mathrm{~g} / \mathrm{dL}$ following dosing or the rate of $\mathrm{Hb}$ level increase was $>1 \mathrm{~g} / \mathrm{dL}$ over a 2 week period. Combined, the number of participants that experienced a rapid increase in $\mathrm{Hb}$ level and subsequent study withdrawal was 21 of $70(30 \%)$ and 8 of $37(22 \%)$ for the CKD-3/4/5 and CKD-5D groups, respectively. While these withdrawals were suggestive of a robust pharmacodynamic response, the study did not reach the target statistical probability of the specified response rate for the higher doses, i.e., 50 and $100 \mathrm{mg}$, in the CKD-3/4/5 group. Only the $25 \mathrm{mg}$ dose in both groups reached the target statistical probability in this study. Accounting for all reasons for withdrawal, only 30 participants in the CKD-3/4/5 group and 22 participants in the CKD-5D group receiving daprodustat had completed the study. With respect to safety of daprodustat, 35 of 61 participants with CKD-3/4/5 and 15 of 31 participants with CKD-5D experienced adverse events (AEs), of which 15 and 1 were considered by the investigator to be drug-related respectively. Nausea was the most common drug-related $\mathrm{AE}$ in the $\mathrm{CKD}-3 / 4 / 5$ group with 5 of the 6 events occurring with those receiving the $100 \mathrm{mg}$ dose. While no deaths were reported in the study, 2 of 7 participants reported serious AEs in the CKD-3/4/5 group, which were assessed as possibly related and both occurred with participants receiving $100 \mathrm{mg}$ doses; none of the reported serious AEs for the 3 participants in the CKD-5D group were considered related to daprodustat.

\section{Exploratory therapeutic indications investigated}

Management of claudication-limited peripheral artery disease: It has been hypothesized that modulating oxygen delivery in patients with peripheral artery disease (PAD) could play a critical role in the treatment of ischemic diseases (NCT 01673555) [19]. Preclinical data suggests that patients with ischemic diseases have reduced HIF activity. This multicentered, randomized placebo-controlled study tested whether administration of daprodustat as a $300 \mathrm{mg}$ single dose followed by a $15 \mathrm{mg}$ daily dose over the course of 2 weeks would affect performance measures in these patients. PK/PD studies were evaluated pre-and post-dosing and a muscle biopsy was performed to assess changes in concentration of HIF gene expression in the muscle. Participants over 40 years old with PAD, defined as having an anklebrachial index $(\mathrm{ABI}) \leq 0.90$ in at least 1 leg at rest for at least 3 months were eligible for the study. Forty-six patients were recruited with no differences in baseline characteristics between active and controlled groups. The most common adverse events seen in the daprodustat group were pain in the extremity, diarrhea, dyspepsia, upper respiratory infections, and fatigue. Eight percent of patients in the daprodustat experienced SAEs of arrhythmias, congestive heart failure, chronic obstructive pulmonary disease, or hyponatremia. There were no differences in improvement in the 6 minute walk test or the bilateral heel rise test. Per PK analysis there was an increase in EPO after the first dose, but there were no differences in EPO levels on subsequent doses compared to placebo. There were increases in $\mathrm{Hb}$ after the subsequent doses of daprodustat were administered. Compared to the placebo group, participants in the daprodustat group exhibited decreases in total cholesterol. For the participants who underwent muscle biopsy, there were no increases in mRNA or proteins of HIF target genes.

The HIF response mechanism was effectively targeted in this patient population, but it did not induce the anticipated clinical response [19]. The lack of response with daprodustat may be due to poor skeletal muscle penetration; however, higher doses would not necessarily resolve this issue without the complications of increased erythropoiesis. 
Page 4 of 5

\section{Safety}

Daprodustat appears to be well tolerated as evidenced by the mild nature and relatively few number of AEs evidenced in the aforementioned phase II trials. The most commonly reported side effect was nausea $[15,16]$. However, due to protocol defined safety criteria, a considerable amount of patients were withdrawn from these studies because of a robust pharmacodynamic $\mathrm{Hb}$ response, indicating a potential for long-term cardiovascular and/or malignant events that have yet to be uncovered given the short time frame for these trials $[15,16]$.

\section{Ongoing Clinical Trials}

Currently, there are 4 phase III clinical trials evaluating the efficacy and safety of daprodustat in populations with varying degrees of CKD, including dialysis dependency and non-dialysis dependency (Table 1) [11]. The trials listed below have parallel assignment study designs against an active comparator, namely darbepoetin alfa, which is an ESA, as well as rhEPO. The primary efficacy endpoints for these trials include measuring mean $\mathrm{Hb}$ between baseline and the efficacy period varying from weeks $40-52$ and weeks $28-5$ and an event driven time to first occurrence of adjudicated major adverse cardiovascular event (MACE) (composite of all-cause mortality, non-fatal myocardial infarction [MI] and non-fatal stroke). Results from composite endpoint in the ASCEND trials will provide clinically meaningful comparative safety data for daprodustat.

\begin{tabular}{|c|c|c|c|c|}
\hline Trial Name & Comparator(s) & ClinicalTrials.gov Identifier & $\begin{array}{l}\text { Estimated } \\
\text { Completion Date }\end{array}$ & Primary \\
\hline $\begin{array}{l}\text { A Study to Evaluate Efficacy and Safety of Daprodustat Compared } \\
\text { to Darbepoetin Alfa in Japanese Hemodialysis (HD) Dependent } \\
\text { Subjects with Anemia Associated with Chronic Kidney Disease } \\
\text { (CKD). }\end{array}$ & Darbepoeitin alfa & NCT02969655 & Jul-18 & \\
\hline $\begin{array}{l}\text { A Study to Evaluate the Efficacy and Safety of Daprodustat } \\
\text { Compared to Recombinant Human Erythropoietin (rhEPO) in } \\
\text { Subjects with Anemia Associated with Chronic Kidney Disease } \\
\text { (CKD) who are Initiating Dialysis. }\end{array}$ & Darbepoeitin alfa & NCT03029208 & Nov-19 & \\
\hline $\begin{array}{l}\text { Anemia Studies in Chronic Kidney Disease: Erythropoiesis via a } \\
\text { Novel Prolyl Hydroxylase Inhibitor Daprodustat Dialysis (ASCEND- } \\
\text { D). }\end{array}$ & rhEPO & NCT02879305 & Apr-20 & \\
\hline $\begin{array}{l}\text { Anemia Studies in Chronic Kidney Disease: Erythropoiesis via a } \\
\text { Novel Prolyl Hydroxylase Inhibitor Daprodustat Non-Dialysis } \\
\text { (ASCEND-ND). }\end{array}$ & Darbepoetin alfa & NCT02876835 & Jan-21 & \\
\hline
\end{tabular}

Table 1: Ongoing phase III daprodustat trials.

\section{Conclusion}

According to the existing body of evidence from the published literature, daprodustat has thus far demonstrated the potential to be an effective alternative for the treatment of anemia in patients with CKD $[15,16,18]$. Daprodustat exhibited dose-dependent EPO and non-EPO response mechanisms for effectively increasing and maintaining $\mathrm{Hb}$ levels. There were no clinically relevant increases in VEGF compared to placebo groups which signifies that the regulation of VEGF by EPO was not influenced by the inhibition of HIF-PH. Although there were no clinically relevant increases in VEGF, a significant percentage of participants were withdrawn due to increases in $\mathrm{Hb} \geq 1 \mathrm{~g} / \mathrm{dL}$ or a $\mathrm{Hb}$ level $>13.5 \mathrm{~g} / \mathrm{dL}$ over a 2 week period as specified by protocol [15]. These robust pharmacodynamic responses over such short time periods may indicate a potential for cardiovascular or carcinogenic side effects with daprodustat when examined over a longer time period. Common side effects such as nausea and dyspepsia were well tolerated and are mostly benign $[15,16]$. Based on the exploratory clinical trial, daprodustat is ineffective for the treatment of PAD, most likely because the drug cannot penetrate the skeletal muscle at safe doses [19]. These data are primarily limited based on the length of the studies and small population sizes. Lastly, there are other HIF-PH inhibitors currently in phase III of development. While these agents share the same mechanism of action they vary with respect to dosing and administration [11]. If the phase III trials for daprodustat reveal positive results, it will be 1 of 4 HIF-PH inhibitors seeking FDA approval for the treatment of anemia in patients with CKD in the upcoming years.

\section{References}

1. Centers for Disease Control and Prevention (2017) Chronic Kidney Disease (CKD) Surveillance System.

2. Stauffer ME, Fan T (2014) Prevalence of anemia in chronic kidney disease in the United States. PLoS ONE 9: e84943.

3. Gupta N, Wish JB (2017) Hypoxia-inducible factor prolyl hydroxylase inhibitors: A potential new treatment for anemia in patients with chronic kidney disease. Am J Kidney Dis 69: 815-826.

4. Onken J, Bregman B, Harrington RA, Morris D, Buerkert J, et al. (2013) Ferric carboxymaltose in patients with iron deficiency anemia and impaired renal function: The REPAIR-IDA trial. Nephrol Dial Transplant 29: 833-842.

5. Epogen (1989). Amgen Inc. Thousand Oaks, CA.

6. Procrit (1989). Amgen Inc. Thousand Oaks, CA.

7. Aranesp (2001). Amgen Inc. Thousand Oaks, CA.

8. Pfeffer MA, Burdmann EA, Chen CY, Cooper ME, Zeeuw DD, et al. (2009) A trial of darbepoetin alfa in type 2 diabetes and chronic kidney disease. N Engl J Med 361: 2019-2032. 
Citation: Becker K, Jones JJ (2017) An Emerging Treatment Alternative for Anemia in CKD Patients: A Review on Daprodustat. J Kidney 3: 152. doi:10.4172/2472-1220.1000152

Page 5 of 5

9. Drueke TB, Locatelli F, Clyne N, Eckardt KU, Macdougall IC, et al. (2006) Normalization of hemoglobin level in patients with chronic kidney disease and anemia. N Engl J Med 355: 2071-2084.

10. GSK group of companies (2017) GSK Press Release.

11. HIF Inhibitor (2017) U.S. National Institutes of Health.

12. Becker K, Saad M (2017) A new approach to the management of anemia: A review on roxadustat. Adv Ther 34: 848-853.

13. Johnson BM, Stier BA, Caltabiano S (2014) Effect of food and gemfibrozil on the pharmacokinetics of the novel prolyl hydroxylase inhibitor GSK1278863. Clin Pharmacol Drug Dev 3: 109-117.

14. Hara K, Takahashi N, Wakamatsu A, Caltabiano S (2015) Pharmacokinetics, pharmacodynamics and safety of single, oral doses of GSK1278863, a novel HIF-prolyl hydroxylase inhibitor, in healthy Japanese and Caucasian subjects. Drug Metab Pharmacokinet 30: 410-418.

15. Brigandi R, Johnson B, Oei C, Westerman M, Olbina G, et al. (2016) A novel hypoxia-inducible factor-prolyl hydroxylase inhibitor (GSK 1278863) for anemia in chronic kidney disease: A 28-day, phase $2 \mathrm{~A}$ randomized trial. Am J Kidney Dis 67: 861-871.
16. Holdstock L, Meadowcroft AM, Maier R, Johnson BM, Jones D, et al. (2016) Four-week studies of oral hypoxia-inducible factor-prolyl hydroxylase inhibitor GSK1278863 for treatment of anemia. J Am Soc Nephrol 27: 1234-1244.

17. Ariazi JL, Duffy KJ, Adams DF, Fitch DM, Luo L, et al. (2017) Discovery and preclinical characterization of GSK1278863 (daprodustat), a small molecule hypoxia inducible factor (HIF)-prolyl hydroxylase inhibitor for anemia. J Pharmacol Exp Ther.

18. Akiwaza T, Tsubakihara Y, Nangaku M, Endo Y, Nakajima H, et al. (2017) Effects of daprodustat, a novel hypoxia-inducible factor prolyl hydroxylase inhibitor on anemia management in Japanese hemodialysis subjects. Am J Nephrol 45: 127-135.

19. Olson E, Demopoulos L, Haws TF, Hu E, Fang Z, et al. (2014) Short-term treatment with a novel HIF-prolyl hydroxylase inhibitor (GSK1278863) failed to improve measures of performance in subjects with claudicationlimited peripheral artery disease. Vasc Med 19: 473-482. 